\title{
Dominance of Metric Correlations in Two-Dimensional Neuronal Cultures Described through a Random Field Ising Model
}

\author{
Lluís Hernández-Navarro, ${ }^{1,2}$ Javier G. Orlandi, ${ }^{1,3}$ Benedetta Cerruti, ${ }^{1,4}$ Eduard Vives, ${ }^{1,2}$ and Jordi Soriano ${ }^{1,2, *}$ \\ ${ }^{1}$ Departament de Física de la Matèria Condensada, Universitat de Barcelona, Barcelona 08028, Catalonia, Spain \\ ${ }^{2}$ Universitat de Barcelona Institute of Complex Systems (UBICS), Barcelona, Catalonia, Spain \\ ${ }^{3}$ Complexity Science Group, Department of Physics and Astronomy, University of Calgary, Calgary, Canada T2N $1 N 4$ \\ ${ }^{4}$ Center for Genomic Science of IIT@SEMM, Istituto Italiano di Tecnologia (IIT), 20139 Milan, Italy
}

(Received 1 June 2016; revised manuscript received 24 March 2017; published 18 May 2017)

\begin{abstract}
We introduce a novel random field Ising model, grounded on experimental observations, to assess the importance of metric correlations in cortical circuits in vitro. Metric correlations arise from both the finite axonal length and the heterogeneity in the spatial arrangement of neurons. The experiments consider the response of neuronal cultures to an external electric stimulation for a gradually weaker connectivity strength between neurons, and in cultures with different spatial configurations. The model can be analytically solved in the metric-free, mean-field scenario. The presence of metric correlations precipitates a strong deviation from the mean field. Null models of the same networks that preserve the distribution of connections recover the mean field. Our results show that metric-inherited correlations in spatial networks dominate the connectivity blueprint, mask the actual distribution of connections, and may emerge as the asset that shapes network dynamics.
\end{abstract}

DOI: 10.1103/PhysRevLett.118.208101

Living neuronal circuits exhibit complex collective behavior that is shaped in great measure by the connectivity among neurons [1]. In the process of associating specific network properties to key dynamical traits it was usually assumed that the spatial constraints of the neuronal circuit could be disregarded. In fact, the combination of physical embedding, spatial organization of the neurons, and wiring cost [2,3] not only prevents a neuron from arbitrarily connecting with any other, but naturally shapes strong correlations and spatially inherited features that can be more important in shaping dynamics than the actual distribution of connections [4]. The importance of these elements and their interrelation is the focus of great attention in the context of spatial networks [5], a framework that analyzes those systems in which nodes and links are embedded in space, and where the physical distance among nodes plays a central role, such as in air transportation, social networks, the Internet, and disease spreading [5-7].

In this Letter we explore the importance of spatial embedding using two-dimensional neuronal cultures [8-10] in which we tune the spatial arrangement of neurons. We frame our observations in the context of an Ising model that can be analytically solved in the metricfree, mean-field approach. This solution provides a reference scenario for the quantification of metric effects and their impact on network behavior.

Our work is grounded on the percolation experiments in neuronal cultures described in Refs. [11-13]. Cultures were prepared by dissociating rat embryonic cortical tissue and plating the cellular population over glass, giving rise to a de novo network that contained both excitatory and inhibitory neurons. For clarity, we show here excitationonly data. Inhibition was fully blocked with $40 \mu \mathrm{M}$ bicuculline. The spatial distribution of neurons varied between a uniform coverage (homogeneous configuration) and a highly localized one (aggregated configuration), and was set by adjusting neuronal adhesion onto the glass substrate [14]. Although neurons covered an area of about $130 \mathrm{~mm}^{2}$, only a region of $0.8 \times 0.7 \mathrm{~mm}^{2}(W \times H)$ was imaged. Details on experimental procedures, possible artifacts associated with the limited imaging window, and the effect of inhibition are provided in the Supplemental Material.

As shown in Fig. 1(a), experiments consisted in obtaining the set of response curves of the neuronal culture upon a series of short, $20 \mathrm{~ms}$ biphasic electric stimulations of gradually higher voltages $V$. Stimulation was delivered through bath electrodes that simultaneously affected all neurons, which responded either according to their sensitivity or in response to other neuronal activations. The neuronal network response to stimulation was quantified through fluorescence calcium imaging [Figs. 1(b)-1(c)], and by counting the fraction of neurons $\phi$ that responded to a given voltage $V$ [18].

The response curves $\phi(V)$ depend on the connectivity between neurons. Synaptic efficacy was reduced by targeting the $\alpha$-amino-3-hydroxy-5-methyl-4-isoxazolepropionic acid (AMPA)-excitatory receptors with the antagonist 6-cyano-7-nitroquinoxaline-2,3-dione (CNQX) [11,12]. For $[\mathrm{CNQX}]=0$ a neuron requires $\tilde{m} \simeq 15$ simultaneous inputs to fire and excite other neurons, a condition known as quorum $[13,20]$. However, more inputs are required as CNQX increases since synaptic strength is weaker, which is expressed as $m=\tilde{m}\left(1+[\mathrm{CNQX}] / K_{d}\right)$, with 
$K_{d}=300 \mathrm{nM}[11,12]$. The variable $m$ represents the control parameter along the disintegration process.

Neuronal coupling is maximum for $[\mathrm{CNQX}]=0$ and $m=\tilde{m}$ [Fig. 1(a)], and thus the stimulation of a small group of neurons suffices to ignite the entire network. The response curve $\phi(V)$ is then characterized by a sharp jump in the fraction of responding neurons, which is associated with the existence of a giant connected component $g$ [11-13], constituted by the largest group of strongly connected neurons. As [CNQX] and $m$ increase, the size of the jump gradually diminishes. At a critical value $m=m_{D}$ (in the range 40-50 for the example shown) a jump is no longer identifiable and $g=0$. At this stage of disintegration the
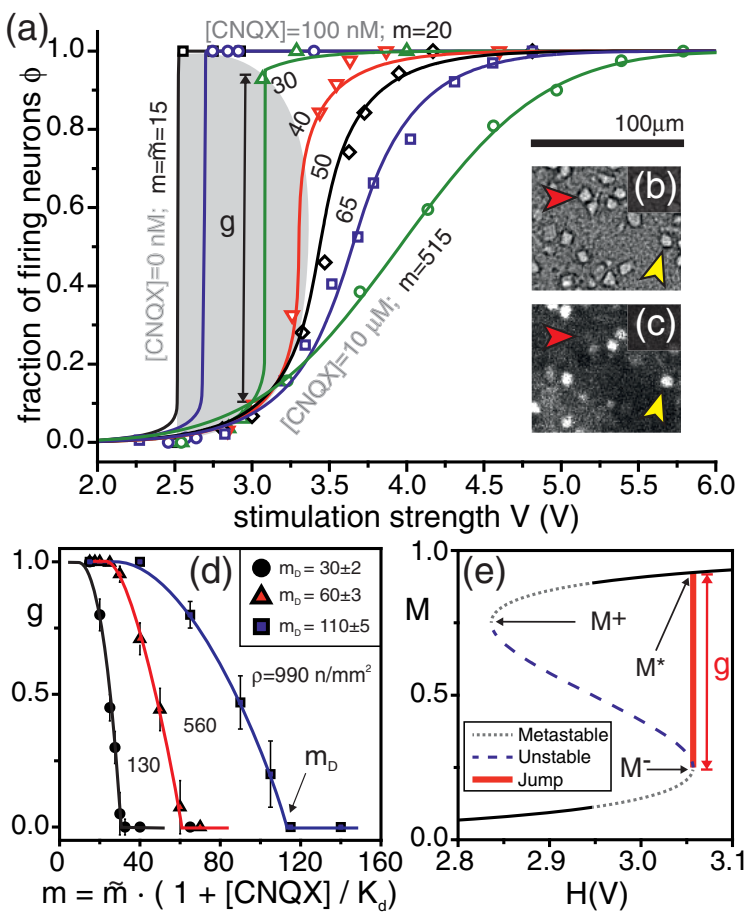

FIG. 1. Experimental results and model construction. (a) Fraction of responding neurons $\phi$ at stimulation voltage $V$ for a homogeneous culture $\left(\rho \simeq 560 \mathrm{n} / \mathrm{mm}^{2}\right.$ ) with only excitatory connections active. From left to right, symbols are experimental data at $[\mathrm{CNQX}]=0,100,300,500,700,1000 \mathrm{nM}$, and $10 \mu \mathrm{M}$. The values of $m=\tilde{m}\left(1+[\mathrm{CNQX}] / k_{d}\right)$ are also indicated, with $\tilde{m}=15$ and $K_{d}=300 \mathrm{nM}$. The grey area illustrates the disintegration of the giant component $g$. The solid lines correspond to the fit of the mean-field approximation to the experiments, and with parameters $H_{0}=4.2 \pm 0.1 \mathrm{~V}, \sigma_{h}=0.80 \pm 0.03 \mathrm{~V}$, and $m_{D}=39 \pm 1$. (b) Bright field image of a region of a neuronal culture. Round objects are neurons. (c) Corresponding fluorescence image. The brightest spots (yellow arrow) are neurons responding to the stimulation. Nonresponding neurons remain darker (red arrow). (d) Disintegration curves $g(m)$ for 3 different neuronal densities $\rho$, each curve showing an average over 4 experimental realizations, and error bars are standard deviation. $m_{D}$ marks the critical value of $m$ at transition. Lines are a guide to the eye. (e) Sketch of the analytical solution in the mean-field approach and the construction of the giant component $g$. network is formed by small isolated clusters, and in the limit $m \rightarrow \infty$ the network is comprised solely of independent neurons. This single-neuron response is well described by an error function, i.e., a Gaussian-distributed neuronal intrinsic variability. This neuronal property does not change with CNQX [21]. Illustrative disintegration curves $g(m)$ for different culture densities $\rho$ are shown in Fig. 1(d), and evince the link between $m_{D}$ and $\rho$.

We cast these observations on a zero-temperature random field Ising model of $N$ spins on a directed network [24-26], with the following features grounded on experiments. First, each neuron presents its own sensitivity to the external field. Second, all connections exhibit the same strength, which decreases with CNQX. And, third, the neuronal network initial activation occurs solely in response to the electrical stimulation. Additionally, we consider that $80 \%$ of the neurons are excitatory and the rest inhibitory [12]. The two populations obey Dales' principle; i.e., excitatory (inhibitory) neurons have solely an excitatory (inhibitory) action.

The Hamiltonian for the neuronal network Ising model (NNIM) then reads

$$
\mathcal{H}=-\sum_{i=1}^{N} s_{i}\left(J \sum_{\left\{j_{E}\right\}_{i}} s_{j}-\gamma \sum_{\left\{j_{I}\right\}_{i}} s_{j}+h_{i}+\tilde{H}\right),
$$

where $s_{i}=0$ or 1 are the spin variables for inactive or active neurons, respectively. $J=J_{0} / m$ is the excitatory exchange energy with a uniform coupling approximation; $J_{0}$ is a constant and $m$ is the experimental control parameter upon disintegration. $\left\{j_{E}\right\}_{i}$ and $\left\{j_{I}\right\}_{i}$ are the set of excitatory and inhibitory input (presynaptic) neighbors of the $i$ th neuron. $\gamma$ is the strength of inhibitory coupling in the network. $h_{i}$ are the Gaussian-distributed local random fields that account for neuronal intrinsic variability. $\tilde{H}$ is the effective external field, defined as $\tilde{H} \equiv H-H_{0}$, with $H_{0}$ a systematic correction. Our observable of interest in the model is the magnetization $M \equiv\langle s\rangle$, i.e., the fraction of active neurons. Table I summarizes the equivalence between the NNIM and experiments. Further details are provided in the Supplemental Material.

The model can be solved in the mean-field approximation (derived in the Supplemental Material), and provides an analytical solution that serves as a metric-free reference

TABLE I. Equivalence between NNIM and experiment.

\begin{tabular}{ll}
\hline \hline NNIM & \multicolumn{1}{c}{ Experiments } \\
\hline Spin variables $s_{i}=\{0,1\}$ & Neuronal state: silent or firing \\
Magnetization $M \equiv\langle s\rangle$ & Fraction of active neurons $\phi$ \\
Coupling strengths $J, \gamma$ & Excitatory \& inhibitory strengths \\
Random fields $h_{i}$ & Neuronal intrinsic variability \\
External field $H$ & Applied voltage $V$ \\
Constant correction $H_{0}$ & Initial inactive state \\
\hline \hline
\end{tabular}


scenario to later quantify the impact of metric correlations. Mean field implies that any neuron effectively experiences the same neighborhood (neuronal type and connectivity) and therefore similar response across the network. We also assume, for simplicity, that all neurons have the same connectivity $\vec{k}$. In the excitation-only scenario, Eq. (1) gives

$$
M=\Phi\left(\sqrt{2 \pi} Q M+\frac{H-H_{0}}{\sigma_{h}}\right),
$$

with $Q \equiv m_{D} / m$. This expression finally provides $g$ in terms of $Q$ as

$$
g+\Phi\left(-\sqrt{\ln Q^{2}}\right)=\Phi\left(\sqrt{2 \pi} Q g-\sqrt{\ln Q^{2}}\right) .
$$

At criticality $\left(m \rightarrow m_{D}\right), g$ scales as $\left(1-m / m_{D}\right)^{1 / 2}$, consistent with the mean field.

The analysis of the model through Eq. (2) provides the set of response curves $\phi(V)$ with $H_{0}, \sigma_{h}$ and $m_{D}$ as parameters, while Eq. (3) provides the disintegration curves $g(m)$ with $m_{D}$ as the only parameter. Additionally, since $Q^{-1} \equiv m / m_{D}$, this equation predicts the existence of a universal disintegration curve when the giant component is plotted as $g\left(m / m_{D}\right)$.

The comparison of the mean-field solution with the experimental results for homogeneous cultures exhibits three interesting features. The first one is that the analytical response curves reflect well the experimental ones [Fig. 1(a)]. The second feature is that the normalized experimental disintegration curves $g\left(m / m_{D}\right)$ neatly collapse (Fig. 2). The $m_{D}$ values are particular of each density, and are obtained by fitting the disintegration data points to the

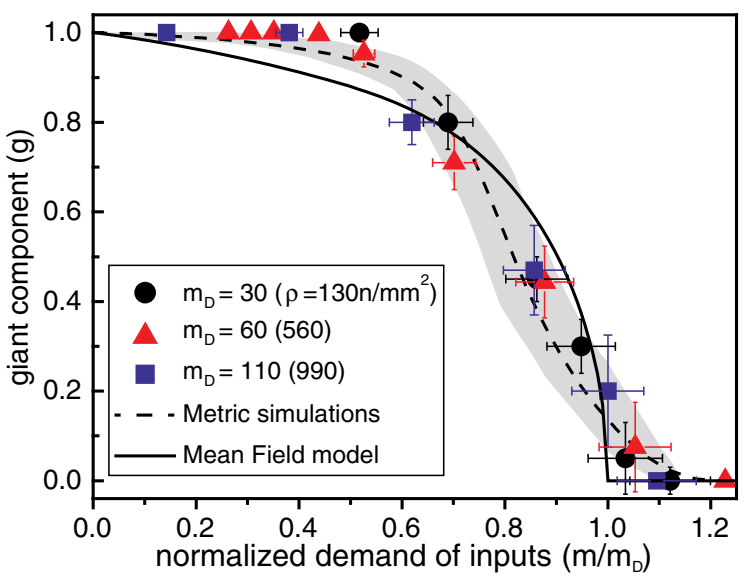

FIG. 2. Comparison between experiments and NNIM. Experimental $g\left(m / m_{D}\right)$ data points for the three densities $\rho$ of Fig. 1(d). Vertical error bars are standard deviation, and horizontal error bars account for the uncertainty in determining $m_{D}$. The solid black line shows the analytical mean-field solution, while the dashed one corresponds to the fit between metric simulations and experimental data. Simulations are an average over 5 network realizations, with the light gray area showing the standard deviation. mean-field solution (see Supplemental Material). And the third feature is that, despite the fairly broad agreement between experiments and the mean field, a clear discrepancy is observed at low $m / m_{D}$, indicating that the mean field is not sufficient to fully capture the behavior of the culture.

We anticipate that this discrepancy is a signature of metric correlations that translate into the loss of the uniform, meanfield neighborhood. We consider that these metric correlations are shaped by two major actors. The first one is the degree of aggregation, $\Lambda$, which accounts for the heterogeneity in the spatial arrangement of neurons. It is computed as the normalized area under the Lorenz curve of neuronal spatial layout (see Supplemental Material). The second one is the relative axonal length, $\delta$, which accounts for the finite maximum connectivity length among neurons relative to the system size. The parameter $\delta$ conceptually reflects the capacity of an arbitrary neuron to connect with any other, and is computed as $\delta=a / L$, where $a$ is the average axonal length and $L$ is the characteristic diameter of the network, given by $L=2 \sqrt{A / \pi}$, with $A$ its total area.

To investigate such a spatially-embedded connectivity scenario we carried out metric numerical simulations of the general NNIM described in Eq. (1) [27]. We incorporated simulations of the corresponding null models, i.e., randomized network analogs that preserved the distribution of connections (see Supplemental Material). We took $\Lambda=$ 0.18 in accordance to our quasihomogeneous cultures, and considered $\delta=0.1$ to fulfill their biological and physical characteristics, namely, $a \simeq 1 \mathrm{~mm}$ and $L \simeq 10 \mathrm{~mm}$. As shown in Fig. 2, metric simulations (dashed line) closely follow the behavior of the experimental data. We note that simulations show a smooth transition due to finite-size effects, as discussed in the Supplemental Material.

To examine the influence of stronger spatial embedding on the $g\left(m / m_{D}\right)$ curves, we extended the numerical simulations to compare networks with contrasting $\Lambda$, namely, $\Lambda=0.18$ (homogeneous configuration) and $\Lambda=0.68$ (aggregated). Averaged $g\left(\mathrm{~m} / \mathrm{m}_{D}\right)$ curves are shown in Fig. 3, with insets depicting illustrative spatial arrangements of neurons and corresponding degree distributions $p(k)$. For clarity, response and disintegration curves for particular realizations of both configurations, as well as the treatment of finite-size effects, are provided in the Supplemental Material. The results in Fig. 3 show that both configurations deviate from the mean field, although the effect is markedly stronger for the aggregated one. Interestingly, the metricinherited $g\left(m / m_{D}\right)$ curves contrast with the corresponding null models, which are almost indistinguishable from one another and neatly follow the mean field.

These simulations show that metric correlations, rather than the distribution of connections, shape in great measure the behavior of the $g\left(m / m_{D}\right)$ curves and bound the range of validity for a mean-field approximation. To provide a measure for the impact of metric correlations in neuronal cultures, we introduce the quantity $\varepsilon$ as the mean-squared 


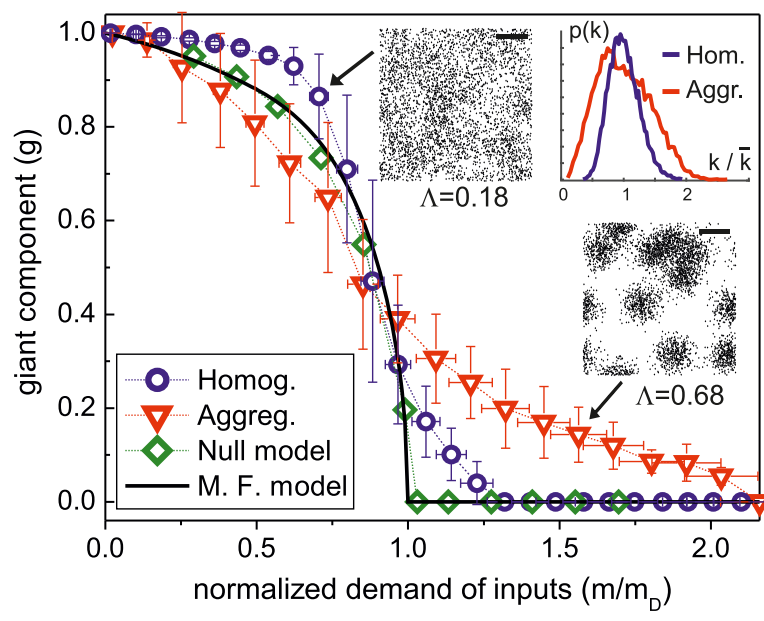

FIG. 3. Simulations of $g\left(m / m_{D}\right)$ curves for contrasting $\Lambda$. Main plot: Normalized disintegration curves for homogeneous (circles) and aggregated (triangles) metric configurations. The null models for both configurations are practically indistinguishable, were averaged out for clarity (diamonds), and neatly follow the mean field. The curves are obtained from the numerical integration of the general NNIM in $10 \times 10 \mathrm{~mm}^{2}$ networks, and the normalization with $m_{D}$ is determined through a fit to the mean-field solution (solid line). Each curve is an average over 10 networks. Vertical error bars denote standard deviation, and horizontal bars account for the uncertainty in the determination of $m_{D}$. The top left and bottom right spatial maps illustrate, respectively, typical aggregated and homogeneous realizations. Scale bars are $1 \mathrm{~mm}$. The top right inset shows the distribution of connections for both realizations, with $\bar{k}=150$.

error between the mean-field solution and the $g\left(m / m_{D}\right)$ curve for a particular network realization at a given degree of aggregation $\Lambda$.

For both experiments and simulations, Fig. 4(a) shows the dependence of $\varepsilon$ on $\Lambda$. The metric-free, null models should exactly follow the mean field and provide $\varepsilon=0 \forall \Lambda$. This occurs, however, for narrow distributions of connections as in Erdös-Rényi graphs [29], or as in our mean-field derivation with $k_{i}=\bar{k} \forall i$. Hence, the presence of a broad distribution of connections in simulations (inset of Fig. 3) leads to a finite $\varepsilon$ and a linear dependence on $\Lambda$, though of small slope. By contrast, metric simulations depict $\varepsilon$ values that are an order of magnitude higher than the corresponding null models, as well as a linear dependence $\varepsilon(\Lambda)$ characterized by a slope 7 times larger. We note that this different behavior arises solely from metric effects that entirely mask the actual distribution of connections. On the other hand, the experiments agree qualitatively well with metric simulations, and evince that spatial constraints in living neuronal networks cannot be disregarded. Examples of homogeneous and aggregated spatial configurations in experiments are provided in Figs. 4(b)-4(c).

In the simulations presented here we considered $\delta=0.1$ in accordance with the experiments. Since $\delta$ portrays a correlation length across the culture, its value is central (see
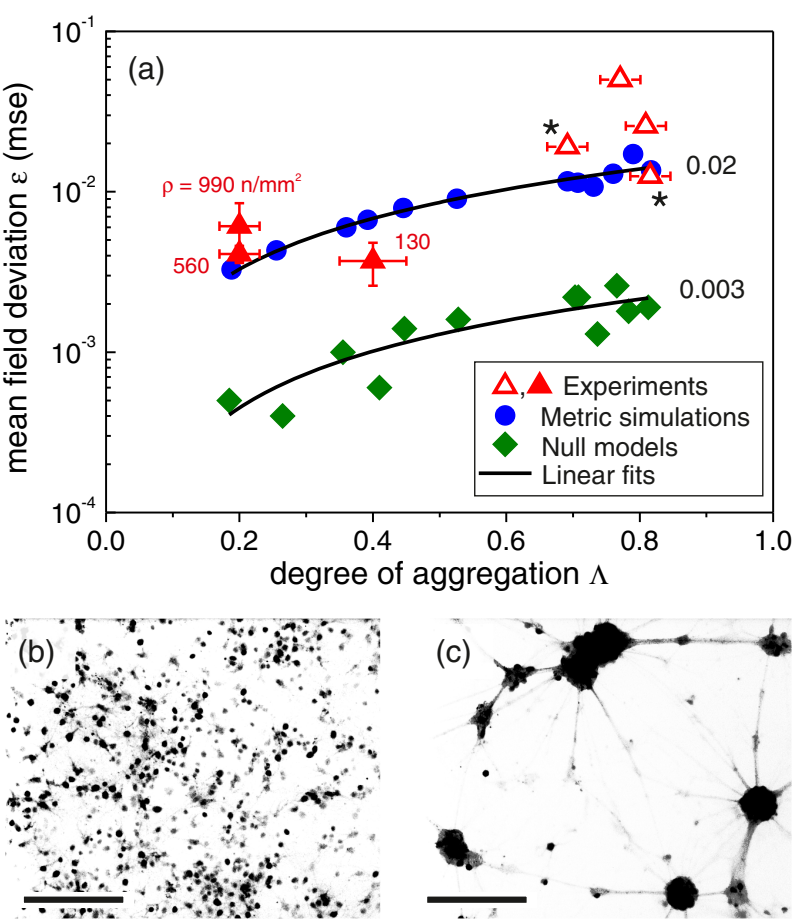

FIG. 4. Deviation from the mean field due to metric correlations. (a) Log-linear representation of $\varepsilon(\Lambda)$ for different network blueprints. Each simulation corresponds to a particular network realization. Solid triangles correspond to the data shown in Fig. 1(d) and Fig. 2, each point averaged over 4 cultures. Vertical error bars are standard deviation. Open triangles are individual cultures. Horizontal error bars reflect the uncertainty in measuring $\Lambda$. Curves are linear fits, with the accompanying value of the slope. Regression coefficients for null models and metric networks are, respectively, $r=0.90$ and $r=0.84$. The $g(m)$ curves for the points marked with * are shown in the Supplemental Material. (b) Highly contrasted image of a homogeneous culture, $\Lambda \simeq 0.2$. (c) Corresponding image for a strongly aggregated culture, $\Lambda \simeq 0.6$. Scale bars are $250 \mu \mathrm{m}$.

Supplemental Material, and Figs. S.13-S.14). For $\delta \rightarrow \infty$ all neurons are interconnected, giving rise to the strictly mean-field scenario, independently, on $\Lambda$. For $\delta \simeq 1$ the network is at the edge of total connectivity and the mean field holds well, although with discrepancies modulated through $\Lambda$. The higher $\Lambda$, the larger the deviation from the mean field. In these conditions, the transition point $m_{D}$ can be assessed by analyzing $g\left(m / m_{D}\right)$ curves and fitting them to the universal mean-field behavior. Percolation studies in inhibition-blocked networks provided $m_{D} \simeq \bar{k} p_{E}$, with $p_{E}$ the fraction of excitatory neurons in the network $[12,13]$. Thus, for those networks sufficiently close to the mean field, our analysis allows for an accurate inference of the network average connectivity $\bar{k}$ without the need of a detailed exploration of the transition point.

At the limit $\delta \rightarrow 0$, the average axonal length is much shorter than the system size, giving rise to networks with strong locality. Here, metric effects are already noticeable 
in homogeneous configurations, and remarkably strong in aggregated ones. For the extreme case of both $\delta \rightarrow 0$ and $\Lambda \rightarrow 1$ the network switches towards a scenario of networks of networks in which the connectivity is markedly distinct at different scales (see Fig. S.10 of the Supplemental Material). This hierarchical structure would shape a system in which the determination of $m_{D}$, and therefore $\bar{k}$, is scale dependent.

The above $\{\delta, \Lambda\}$ regimes characterize the crossover that separates metric-free scenarios from metric-dominated ones. This crossover was also suggested by others [29-31]; however, they considered $\Lambda \rightarrow 0$ (lattice-like networks), disregarding the crucial role of $\Lambda$.

We conjecture that metric effects overshadow the distribution of connections to such an extreme that they mold the dynamic attributes of the network. Such an idea is supported by experimental results in neuronal cultures $[10,32]$. For instance, the analysis of activity fronts in homogeneous cultures similar to ours $(\delta \simeq 0.1, \Lambda \simeq 0.2)$ revealed that metric correlations provide amplification mechanisms for the fronts to spontaneously emerge [10]. The initiation of the fronts was highly localized in space and propagated in a circular manner throughout the network. At the other extreme, in cultures with $\Lambda \gtrsim 0.6$ [Fig. 4(c)], the dynamics was characterized by a fast synchronous firing at the scale of the neuronal aggregates combined with a slower sequential activation of small groups of aggregates at the system's scale $[15,33]$.

Our work offers the possibility to predict the dynamical complexity of spatial networks given $\delta$ and $\Lambda$. These spatial features are not only important to comprehend the structure-function interplay in living neuronal networks, but also in all those systems where spatial embedding shapes highorder network features such as navigation or robustness.

We thank S. Rüdiger and E. Moses for useful conversations. This work was supported by the Spanish Ministerio de Economia y Competitividad through Projects No. FIS2013-41144-P, No. MAT2013-40590-P, and No. MAT2015-69-777-REDT; and by the Generalitat de Catalunya through Grants No. 2014-SGR-878 and No. 2014FI-B00721.

*jordi.soriano@ub.edu

[1] O. Sporns, D. R. Chialvo, M. Kaiser, and C. C. Hilgetag, Trends Cognit. Sci. 8, 418 (2004).

[2] E. Bullmore and O. Sporns, Nat. Rev. Neurosci. 13, 336 (2012).

[3] Y. Chen, S. Wang, C. C. Hilgetag, and C. Zhou, PLoS Comput. Biol. 9, e1002937 (2013).

[4] S. Horvát, R. Gămănuţ, M. Ercsey-Ravasz, L. Magrou, B. Gămănuţ, D. C. Van Essen, A. Burkhalter, K. Knoblauch, Z. Toroczkai, and H. Kennedy, PLoS Biol. 14, e1002512 (2016).

[5] M. Barthlemy, Phys. Rep. 499, 1 (2011).
[6] G. Bianconi, P. Pin, and M. Marsili, Proc. Natl. Acad. Sci. U.S.A. 106, 11433 (2009).

[7] D. Brockmann and D. Helbing, Science 342, 1337 (2013).

[8] J.-P. Eckmann, O. Feinerman, L. Gruendlinger, E. Moses, J. Soriano, and T. Tlusty, Phys. Rep. 449, 54 (2007).

[9] E. Tibau, M. Valencia, and J. Soriano, Front. Neural Circuits 7, 204 (2013).

[10] J. G. Orlandi, J. Soriano, E. Alvarez-Lacalle, S. Teller, and J. Casademunt, Nat. Phys. 9, 582 (2013).

[11] I. Breskin, J. Soriano, E. Moses, and T. Tlusty, Phys. Rev. Lett. 97, 188102 (2006).

[12] J. Soriano, M. R. Martínez, T. Tlusty, and E. Moses, Proc. Natl. Acad. Sci. U.S.A. 105, 13758 (2008).

[13] O. Cohen, A. Keselman, E. Moses, M. R. Martínez, J. Soriano, and T. Tlusty, Europhys. Lett. 89, 18008 (2010).

[14] See Supplemental Material at http://link.aps.org/ supplemental/10.1103/PhysRevLett.118.208101 for details on culture preparation, which includes Refs. [15-17].

[15] S. Teller, C. Granell, M. De Domenico, J. Soriano, S. Gómez, and A. Arenas, PLoS Comput. Biol. 10, e1003796 (2014).

[16] R. Segev, M. Benveniste, Y. Shapira, and E. Ben-Jacob, Phys. Rev. Lett. 90, 168101 (2003).

[17] D. de Santos-Sierra, I. Sendiña-Nadal, I. Leyva, J. A. Almendral, A. Ayali, S. Anava, C. Sánchez-Ávila, and S. Boccaletti, Cytometry 87, 513 (2015).

[18] See Supplemental Material at http://link.aps.org/supplemental/ 10.1103/PhysRevLett.118.208101 for the quantification of active neurons, which includes Refs. [11, 12, 19].

[19] T. Sasaki, N. Takahashi, N. Matsuki, and Y. Ikegaya, J. Neurophysiol. 100, 1668 (2008).

[20] S. Métens, P. Monceau, R. Renault, and S. Bottani, Phys. Rev. E 93, 032112 (2016).

[21] See Supplemental Material at http://link.aps.org/supplemental/ 10.1103/PhysRevLett.118.208101 for the insensitivity of neuronal excitability to CNQX, which includes Refs. [22,23].

[22] K. A. Yamada, J. M. Dubinsky, and S. M. Rothman, J. Neurosci. 9, 3230 (1989).

[23] R. Vergara, C. Rick, S. Hernández-López, J. A. Laville, J. N. Guzman, E. Galarraga, D. J. Surmeier, and J. Bargas, J. Physiol. 553, 169 (2003).

[24] J. P. Sethna, K. Dahmen, S. Kartha, J. A. Krumhansl, B. W. Roberts, and J. D. Shore, Phys. Rev. Lett. 70, 3347 (1993).

[25] D. Dhar, P. Shukla, and J. P. Sethna, J. Phys. A 30, 5259 (1997).

[26] X. Illa, P. Shukla, and E. Vives, Phys. Rev. B 73, 092414 (2006).

[27] See Supplemental Material at http://link.aps.org/ supplemental/10.1103/PhysRevLett.118.208101 for details on the numerical simulations, which includes Ref. [28].

[28] F. Salvat-Pujol, E. Vives, and M.-L. Rosinberg, Phys. Rev. E 79, 061116 (2009).

[29] D. Li, G. Li, K. Kosmidis, H. E. Stanley, A. Bunde, and S. Havlin, Europhys. Lett. 93, 68004 (2011).

[30] T. Tlusty and J.-P. Eckmann, J. Phys. A 42, 205004 (2009).

[31] C. Schmeltzer, J. Soriano, I. M. Sokolov, and S. Rüdiger, Phys. Rev. E 89, 012116 (2014).

[32] S. Okujeni, S. Kandler, and U. Egert, J. Neurosci. 37, 3972 (2017)

[33] S. Teller, I. B. Tahirbegi, M. Mir, J. Samitier, and J. Soriano, Sci. Rep. 5, 17261 (2015) 